\title{
The COP9 signalosome subunit 6 (CSN6): a potential oncogene
}

\author{
Shang-Nuan Zhang ${ }^{1}$, Dong-Sheng Pei ${ }^{1 *}$ and Jun-Nian Zheng ${ }^{1,2^{*}}$
}

\begin{abstract}
CSN6 is one subunit of the constitutive photomorphogenesis 9 (COP9) signalosome (CSN), which is an evolutionarily conserved multiprotein complex found in plants and animals and originally described as a repressor of light-dependent growth and transcription in Arabidopsis. CSN is homologous to the 195 lid subcomplex of the 265 proteasome, thus it has been postulated to be a regulator of the ubiquitin-proteasome pathway. In mammalian cells, it consists of eight subunits (CSN1-CSN8). Among the CSN subunits, CSN5 and CSN6 are the only two that each contains an MPN (Mpr1p and Pad1p N-terminal) domain. The deneddylating activity of an MPN domain toward cullin-RING ubiquitin ligases (CRL) may coordinate CRL-mediated ubiquitination activity. More and more studies about CSN6 are emerging, and its overexpression is found in many types of cancers. Evidence has shown that CSN6 is a molecule platform between protein degradation and signal transduction. Here, we provide a summary of human CSN6, especially its roles in cancer, hoping that it can lay the groundwork for cancer prevention or therapy.
\end{abstract}

Keywords: CSN, CSN6, Cancer, Ubiquitination, Deneddylation

\section{Introduction}

The COP9 signalosome, generally named CSN, is an evolutionarily conserved multiprotein complex existing in all eukaryotes. It consists of eight subunits termed CSN1-CSN8 [1]. Among the CSN subunits, CSN6 and CSN5 are the only two subunits that each share an MPN (Mpr1p and Pad1p N-terminal) domain, while other subunits contain a PCI domain, which may serve as a structure scaffold in the assembly of the COP9 signalosome [2-4].

The complex was originally described as a repressor of light-dependent growth in Arabidopsis by Deng and his collaborators [5]. CSN has diverse functions in cellular and developmental processes, including cell cycle control, signal transduction, transcriptional activation [6,7] and tumorigenesis $[8,9]$. The most and best studied function is regulation of ubiquitin-mediated protein degradation [10-13]. The function is fulfilled by removal of Nedd8/ Rub1 (an ubiquitin-like molecule) from the cullin subunit of cullin-containing E3 ligases. And the deneddylation

\footnotetext{
* Correspondence: dspei@xzmc.edu.cn; jnzheng@xzmc.edu.cn
'Jiangsu Key Laboratory of Biological Cancer Therapy, Xuzhou Medical

* Correspondence: dspei@xzmc.edu.cn; jnzheng@xzmc.edu.cn
'Jiangsu Key Laboratory of Biological Cancer Therapy, Xuzhou Medical College, 84 West Huai-hai Road, Xuzhou, Jiangsu, P.R. China

${ }^{2}$ Center of Clinical Oncology, Affiliated Hospital of Xuzhou Medical College, Xuzhou 221002, China
}

activity toward cullins is necessary for maintaining the stability and the sustained activity of cullin-RING E3 ligases (CRLs) in vivo, allowing the ligases to polyubiquitinate a large number of substrates that are targeted by the ubiquitin-proteasome system [14-17]. Since many key oncogene and tumor suppressor products such as p27

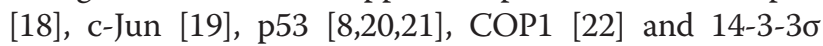
[22] are degraded via the ubiquitin-proteasome pathway, it is conceivable that COP9 plays a significant role in cancer.

As a subunit of COP9 signalosome complex, CSN6 is found to be overexpressed in many types of cancers $[8,23]$, linking it to oncogenic activity. Therefore, in this review, we provide an overview of the role of CSN6 in cancer and summarize recent findings that highlight the novel roles of CSN6 in cellular and developmental processes, suggesting that CSN6 is a promising therapeutic target in combating human cancers.

\section{CSN6 plays an important role in structural integrity of the CSN complex}

The CSN is $\sim 450 \mathrm{kDa}$ in mass and comprises eight core subunits called CSN1-CSN8, in order of descending subunit size [24]. Then, how is this important complex organized structurally? 
It has been shown that the complex is composed of two symmetrical modules, CSN1/2/3/8 and CSN4/5/6/7, connected by interactions between CSN1 and CSN6 [25]. As mentioned above, CSN6 and CSN5 are the only two subunits that each share an MPN (Mpr1p and Pad1p N-terminal) domain. Although CSN6 and CSN5 both contain the MPN domain, evidence has revealed that there are two types of MPN domains: one, like CSN5 with JAMM (Jab1/MPN/Mov34) motif, has the metalloprotease activity while another, the same as CSN6 without JAMM motif, has no related isopeptidase activity, which is probably involved in protein-protein interaction and in protein stability [26]. In fact, CSN6 contains an N-terminal MPN domain and a newly identified S6CD domain in the carboxyl half of the protein [27]. The MPN domain is responsible for the interaction with CSN5, and the S6CD-containing C-terminal half of CSN6 is in association with CSN4 and CSN7, suggesting that CSN6 is a core protein in the CSN4/5/6/7 subcomplex [28]. Moreover, earlier genetic studies in Arabidopsis showed that complete depletion of CSN6 resulted in loss of the entire CSN complex [29]. Therefore, CSN6 is essential for CSN assembly.

\section{CSN6 is involved in proteasome-mediated protein degradation}

As mentioned above, COP9 coordinates CRL-mediated ubiquitination activity through its associated deneddylation activity toward the cullin subunit of cullincontaining E3 ligases. The deneddylation activity localizes to the JAMM (JAB1/MPN/Mov34) motif of CSN5 [30]. However, CSN5 alone has no metalloprotease activity unless it is associated with other subunits. Thus the deneddylation activity also requires the integrity of the CSN complex, which needs CSN6. Moreover, it has been shown that CSN6 makes a contribution to the binding of CSN and E3 ligases [30,31]. Recent study further demonstrates that the C-terminal region of CSN6 is necessary and sufficient for CSN complex integrity and recruitment of cullins to the CSN complex [27]. Taken together, CSN6 plays a pivotal role in proteasome-mediated protein degradation via regulating E3 ligases such as MDM2 [8] and COP1 [22].

\section{Roles of CSN6 in tumor CSN6 is overexpressed in cancer}

The human cancer patient transcriptomic data sets from Oncomine and Gene Expression Omnibus analyzed using Oncomine analysis tools and Nexus expression 2.0 reveal that many types of cancer have CSN6 overexpression, such as glioblastoma, breast cancer, myeloma, leukemia [23]. Zhao et al [8]. used the System for Integrative Genomic Microarray Analysis (SIGMA [32]) to evaluate genetic loss or gain of CSN6 (located at 7q22.1) using data from the British Columbia Cancer Agency Research (BCCRC) SMRT arrays, and found that a substantial percentage of samples (breast cancer cell lines and other types of cancer cells) had amplification of the CSN6 genomic region. And then they used quantitative PCR to confirm the gene amplification of CSN6 in breast cancer samples experimentally. Amplification of CSN6 was detected in a high percentage of breast cancer samples, and there was a positive correlation between CSN6 gene copy number and tumor size. Also, by comparing malignant follicular thyroid carcinomas with benign thyroid lesions (follicular adenomas, adenomatous nodules, and multinodular goiters) and normal thyroid tissue, they found that the carcinomas expressed higher levels of CSN6 than did benign lesions and tissues. These results suggest that CSN6 is overexpressed in cancer and is not restricted to a few specific types or cases of cancer.

\section{Physiological significance of CSN6 overexpression in cancer}

CSN6 is overexpressed in many types of cancer $[8,23]$, linking it to oncogenic activity. However, detailed mechanisms through which CSN6 contributes toward carcinogenesis/tumor development remain unclear. Previously, human CSN6 was identified to interact with the human immunodeficiency virus 1 accessory protein $\mathrm{Vpr}$ (also called hVIP for Vpr interactive protein) and found to be involved in the $\mathrm{G} 2 / \mathrm{M}$ phase transition of the cell cycle and cell proliferation [33]. Recently, more and more studies about CSN6 focus on the signaling pathways in which it is involved during carcinogenesis/tumor progression.

\section{CSN6-MDM2-p53 axis}

As a RING domain-containing E3 ubiquitin ligase, MDM2 can ubiquitin tumor suppressor p53 at several lysine residues, which causes p53 degradation. It can also degrade itself by autoubiquitination [34,35]. Overexpression of MDM2 is found in a wide variety of human tumors [36]. Zhao et al [8]. studied the expression of MDM2 and CSN6 in matched normal and cancerous breast tissues, and found that CSN6 was concomitantly overexpressed with MDM2 in human breast cancer tissues. Mechanism studies indicated that CSN6 prevented MDM2 autoubiquitination at lysine 364, resulting in stabilization of MDM2 and degradation of p53. Moreover, CSN6 couldn't induce p53 degradation in Mdm2-null mouse embryonic fibroblasts, suggesting that CSN6mediated degradation of p53 is MDM2 dependent. Mice in which CSN6 was deleted died early in embryogenesis, which could be rescued by concomitant loss of p53. Mice heterozygous for CSN6 (CSN6+/-) exposed to high doses of $\gamma$-irradiation (IR) showed more apoptosis due to an increased p53 activity. And loss of expression of CSN6 could attenuate carcinogenesis/tumor progression in response to DNA damage, which is known to be 
impeded by p53. These results suggest that CSN6 is an oncogene with positive activity toward MDM2 and plays a significant role in DNA damage-associated apoptosis and tumorigenesis through MDM2-p53 signaling pathway.

\section{CSN6-COP1 axis}

As another E3 ubiquitin ligase for p53, COP1 targets p53 for degradation by ubiquitin-dependent proteasome system, independently of MDM2 or Pirh2, which are known to interact with and negatively regulate $\mathrm{p} 53$, thereby maintaining p53 at low levels in unstressed cells and inhibiting

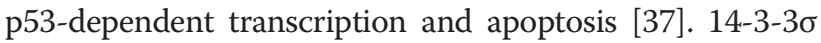
is a gene upregulated by $\mathrm{p} 53$ and has a positive feedback effect on p53 in response to DNA damage. It has been found to be frequently lost or decreased in various human cancers and functions as a potential tumor suppressor [38-40]. Recently, CSN6 was found to interact with COP1 and be involved in 14-3-3 $\sigma$ ubiquitin-mediated degradation [22]. Co-IP shows that CSN6 associates with COP1 endogenously and in vivo binding assay confirms that CSN6 directly binds to COP1 [22]. CSN6 stabilizes COP1 through reducing COP1 self-ubiquitination and decelerating COP1's turnover rate [22]. The CSN6-COP1 axis has following physiological significance possibly: (1) CSN6 stabilizing COP1 directly enhances COP1-mediated p53 ubiquitination and degradation. (2) CSN6-COP1 axis causes 14-3-3 $\sigma$ degradation, which on the one hand, can block 14-3-3 $\sigma$ 's positive effect on p53 stability, on the other hand, activates Akt and promotes Akt-mediated cell survival.

\section{CSN6-p57 axis}

Recently, it is shown that CDK inhibitor (CDI) p57 $7^{\text {kip2 }}$ is a new target of CSN6 [41]. The p57 ${ }^{\mathrm{Kip} 2}$ protein (abbreviated as $\mathrm{p} 57$ ) is a member of $\mathrm{p} 21^{\mathrm{Cip} 1} / \mathrm{p} 27^{\mathrm{Kip} 1}$ CDI family, sharing similar sequence with $\mathrm{p} 27^{\mathrm{Kip} 1}$, also known as CDKN1C. Its overexpression causes a complete cell cycle arrest in G1 phase [42]. Decreased expression of p57 has been found in many types of cancer, including bladder carcinoma, gastric cancer and pancreatic cancer [43]. Stabilization of p57 is essential for the maintenance of its tumor suppressor function. Therefore, deregulation of proteins that affect p57 protein stability is expected to have an impact on human tumorigenesis. It's well known that degradation of p57 is dependent on ubiquitylation, which is mediated by Skp2, an important component of Skp1/Cul1/F-box (SCF)-type E3 ubiquitin ligase [44]. Chen et al [41]. found that CSN6 was involved in p57 downregulation and increased the ubiquitination level of p57 in a dose-dependent manner. Mechanism studies show that CSN6 interacts with p57 and Skp2 through its C-terminal domain, which, in turn, promotes Skp2mediated protein ubiquitination of $\mathrm{p} 57$, thereby decreasing the steady-state expression of p57. Significantly,
CSN6 overexpression antagonized p57-mediated cell proliferation inhibition, G1 arrest and cell transformation suppression. Moreover, high expression levels of CSN6 and low expression levels of p57 correlated with poor overall survival in human tumor samples. Thereby, the CSN6-p57 link will be an important molecular target for cancer therapy and intervention [41].

\section{HER2-Akt-CSN6 axis}

CSN6 plays an important role in protein degradation, however, the molecular signals in regulating CSN6 activity are largely unknown. It is well known that HER2Akt is critical in regulating p53 activity through MDM2 [45]. Recent study has shown that CSN6 is pivotal in regulating MDM2 to destabilize p53 [8]. Based on these studies, Lee's laboratory further found that HER2-Akt axis is linked to CSN6 regulation, and that Akt is a positive regulator of CSN6 [46]. Akt is able to associate with CSN6 and phosphorylate it at Ser60, which can reduce the ubiquitin-mediated protein degradation and turnover rate of CSN6, thereby increasing steady-state expression of CSN6. Furthermore, Akt's positive impact on CSN6 overexpression results in p53 degradation, cell transformation and DNA damage [46].

\section{CSN6 cleavage during apoptosis can regulate CSN-mediated deneddylation}

In addition, few studies point out that CSN6 may participate in apoptosis process. For example, CSN6 can interact with amino-terminal caspase recruitment domain (CARD) of Nod1, a cytoplasmic protein that belongs to the Nod/ NLR/CATERPILLER protein family and whose activation is involved in apoptotic pathways. During Nod1-induced apoptosis process, CSN6 is cleaved by recombinant caspase 8, suggesting that CSN6 is a direct target of caspase 8 [47]. However, how CSN6 is cleaved and the significance of CSN6 cleavage is still unclear. Later, in vitro and in vivo experiments further indicate that CSN6 is cleaved during apoptosis by activated caspases, most effectively by active caspase 3. And CSN6 cleavage is followed by cleavage of Rbx1, a component of CRL, thereby resulting in activation of CSN-mediated deneddylation and inactivation of CRLs [48]. These data demonstrate that CSN-mediated deneddylation can be regulated by active caspases and that the CSN6 executes a specific function during the apoptotic process.

\section{Concluding remarks}

In this review, we have discussed the contribution of CSN6 to COP9 signalosome structurally and functionally, particularly focusing on roles of CSN6 in carcinogenesis/ tumor development. As a subunit of COP9 signalosome, the roles of CSN6 in cancer could be linked to its involvement in ubiquitin-mediated protein degradation. However, 
the precise mechanism underlying its roles in regulating Cullin-RING E3 ligases remain to be fully elucidated.

It has been confirmed that deneddylation activity localizes to the JAMM (JAB1/MPN/Mov34) motif of CSN5 [30]. Nonetheless, the MPN domain of CSN6 is responsible for protein-protein interaction, while the newly identified S6CD domain in the carboxyl half of CSN6 is necessary and sufficient for CSN complex integrity and recruitment of cullins to the CSN complex [27], which is needed for deneddylation activity. Therefore, it seems that CSN6 executing its function is based on its effects on the whole CSN complex.

Recent findings about CSN6 mainly focus on its involvement in signaling pathways during tumorigenesis. For example, CSN6 connects HER2-Akt pathway with MDM2-p53 pathway [49], thereby regulating the expression of p53, a crucial tumor suppressor well known to us. In addition, CSN6 can stabilize COP1 and target substrates of COP1, such as 14-3-3 $\sigma$ and p53. Recently, p57, a new target of CSN6, is found to be regulated by CSN6 through Skp2. However, little is known about its upstream regulators. So far, few upstream regulators of CSN6 have been mentioned. For instance, Akt can positively regulate CSN6 by phosphorylation. Caspases also cleave CSN6 during apoptosis. Thus it is necessary to discover new regulators and targets of CSN6. As for the phosphorylation event, Akt can phosphorylate CSN6 and MDM2. However, could it regulate other p53 ubiquitin ligases, such as COP1, MDMx [50,51], to participate in p53 degradation? Is it also regulated by DNA damage? Could the upregulation of p53 in response to DNA damage have effect on CSN6 in turn?

In conclusion, CSN6 takes an active part in carcinogenesis/tumor development, suggesting its oncogene activity. Further investigations to understand the functions of CSN6 and its roles in cancer should be performed, thus significantly accelerating the development of a novel therapeutic strategy for various types of cancer.

\section{Competing interests}

The authors declare that they have no competing interests.

\section{Authors' contributions}

SNZ collected and reviewed the literature and wrote the manuscript. DSP and JNZ corrected and revised the manuscript. All authors read and approved the final manuscript.

\section{Acknowledgments}

This work was supported by the National Natural Science Foundation of China (No. 81372172) and the key project of the Education Department of China (212062).

Received: 17 October 2013 Accepted: 18 November 2013 Published: 28 November 2013

\section{References}

1. Deng XW, Dubiel W, Wei N, Hofmann K, Mundt K: Unified nomenclature for the COP9 signalosome and its subunits: an essential regulator of development. Trends Genet 2000, 16:289.
2. Aravind $L$, Ponting CP: Homologues of 265 proteasome subunits are regulators of transcription and translation. Protein Sci 1998, 7:1250-1254.

3. Glickman MH, Rubin DM, Coux O, Wefes I, Pfeifer G, Cjeka Z, Baumeister W, Fried VA, Finley D: A subcomplex of the proteasome regulatory particle required for ubiquitin-conjugate degradation and related to the COP9-signalosome and elF3. Cell 1998, 94:615-623.

4. Hofmann K, Bucher P: The PCl domain: a common theme in three multiprotein complexes. Trends Biochem Sci 1998, 23:204-205.

5. Wei N, Chamovitz DA, Deng XW: Arabidopsis Cop9 is a component of a novel signaling complex mediating light control of development. Cell 1994, 78:117-124.

6. Wei N, Deng XW: Making sense of the COP9 signalosome. A regulatory protein complex conserved from Arabidopsis to human. Trends Genet 1999, 15:98-103.

7. Seeger M, Kraft R, Ferrell K, Bech-Otschir D, Dumdey R, Schade R, Gordon C, Naumann $M$, Dubiel W: A novel protein complex involved in signal transduction possessing similarities to $26 \mathrm{~S}$ proteasome subunits. FASEB J 1998, 12:469-478.

8. Zhao RY, Yeung SCJ, Chen JA, Iwakuma T, Su CH, Chen B, Qu CJ, Zhang FM, Chen YT, Lin YL, et al: Subunit 6 of the COP9 signalosome promotes tumorigenesis in mice through stabilization of MDM2 and is upregulated in human cancers. J Clin Invest 2011, 121:851-865.

9. Richardson KS, Zundel W: The emerging role of the COP9 signalosome in cancer. Mol Cancer Res 2005, 3:645-653.

10. Schwechheimer C, Deng XW: COP9 signalosome revisited: a novel mediator of protein degradation. Trends Cell Biol 2001, 11:420-426.

11. Bech-Otschir D, Seeger M, Dubiel W: The COP9 signalosome: at the interface between signal transduction and ubiquitin-dependent proteolysis. J Cell Sci 2002, 115:467-473.

12. Cope GA, Deshaies RJ: COP9 signalosome: a multifunctional regulator of SCF and other cullin-based ubiquitin ligases. Cell 2003, 114:663-671.

13. Wolf DA, Zhou C, Wee S: The COP9 signalosome: an assembly and maintenance platform for cullin ubiquitin ligases? Nat Cell Biol 2003, 5:1029-1033.

14. Hotton SK, Callis J: Regulation of cullin RING ligases. Annu Rev Plant Biol 2008, 59:467-489.

15. Peth A, Berndt C, Henke W, Dubiel W: Downregulation of COP9 signalosome subunits differentially affects the CSN complex and target protein stability. BMC Biochem 2007, 8:27.

16. Higa LA, Zhang $H$ : Stealing the spotlight: CUL4-DDB1 ubiquitin ligase docks WD40-repeat proteins to destroy. Cell Div 2007, 2:5

17. Su HB, Huang W, Wang XJ: The COP9 signalosome negatively regulates proteasome proteolytic function and is essential to transcription. Int J Biochem Cell B 2009, 41:615-624.

18. Tomoda K, Kubota Y, Kato J: Degradation of the cyclin-dependent-kinase inhibitor p27Kip1 is instigated by Jab1. Nature 1999, 398:160-165.

19. Naumann M, Bech-Otschir D, Huang XH, Ferrell K, Dubiel W: COP9 signalosome-directed c-Jun activation/stabilization is independent of JNK. J Biol Chem 1999, 274:35297-35300.

20. Bech-Otschir D, Kraft R, Huang XH, Henklein P, Kapelari B, Pollmann C, Dubiel W: COP9 signalosome-specific phosphorylation targets p53 to degradation by the ubiquitin system. Embo J 2001, 20:1630-1639.

21. Zhang XC, Chen J, Su CH, Yang HY, Lee MH: Roles for CSN5 in control of p53/MDM2 activities. J Cell Biochem 2008, 103:1219-1230.

22. Choi HH, Gully C, Su CH, Velazquez-Torres G, Chou PC, Tseng C, Zhao R, Phan L, Shaiken T, Chen J, et al: COP9 signalosome subunit 6 stabilizes COP1, which functions as an E3 ubiquitin ligase for 14-3-3sigma. Oncogene 2011, 30:4791-4801.

23. Lee MH, Zhao R, Phan L, Yeung SC: Roles of COP9 signalosome in cancer Cell cycle 2011, 10:3057-3066.

24. Chamovitz DA, Segal D: JAB1/CSN5 and the COP9 signalosome, a complex situation. EMBO Rep 2001, 2:96-101.

25. Sharon M, Mao H, Boeri Erba E, Stephens E, Zheng N, Robinson CV: Symmetrical modularity of the COP9 signalosome complex suggests its multifunctionality. Structure 2009, 17:31-40.

26. Sanches M, Alves BS, Zanchin NI, Guimaraes BG: The crystal structure of the human Mov34 MPN domain reveals a metal-free dimer. J Mol Biol 2007, 370:846-855.

27. Pick E, Golan A, Zimbler JZ, Guo L, Sharaby Y, Tsuge T, Hofmann K, Wei N: The minimal deneddylase core of the COP9 signalosome excludes the Csn6 MPN- domain. PLoS One 2012, 7:e43980. 
28. Kotiguda GG, Weinberg D, Dessau M, Salvi C, Serino G, Chamovitz DA, Hirsch JA: The organization of a CSN5-containing subcomplex of the COP9 signalosome. J Biol Chem 2012, 287:42031-42041.

29. Gusmaroli G, Figueroa P, Serino G, Deng XW: Role of the MPN subunits in COP9 signalosome assembly and activity, and their regulatory interaction with Arabidopsis Cullin3-based E3 ligases. Plant Cell 2007, 19:564-581.

30. Cope GA, Suh GS, Aravind L, Schwarz SE, Zipursky SL, Koonin EV, Deshaies RJ: Role of predicted metalloprotease motif of Jab1/Csn5 in cleavage of Nedd8 from Cul1. Science 2002, 298:608-611.

31. Lyapina S, Cope G, Shevchenko A, Serino G, Tsuge T, Zhou C, Wolf DA, Wei N, Shevchenko A, Deshaies RJ: Promotion of NEDD-CUL1 conjugate cleavage by COP9 signalosome. Science 2001, 292:1382-1385.

32. Chari R, Lockwood WW, Coe BP, Chu A, Macey D, Thomson A, Davies JJ, MacAulay C, Lam WL: SIGMA: a system for integrative genomic microarray analysis of cancer genomes. BMC Genomics 2006, 7:324

33. Mahalingam S, Ayyavoo V, Patel M, Kieber-Emmons T, Kao GD, Muschel RJ, Weiner DB: HIV-1 Vpr interacts with a human 34-kDa mov34 homologue, a cellular factor linked to the G2/M phase transition of the mammalian cell cycle. Proc Natl Acad Sci USA 1998, 95:3419-3424.

34. Fang $\mathrm{S}$, Jensen JP, Ludwig RL, Vousden $\mathrm{KH}$, Weissman AM: Mdm2 is a RING finger-dependent ubiquitin protein ligase for itself and p53. J Biol Chem 2000, 275:8945-8951.

35. Inuzuka H, Fukushima $H$, Shaik S, Wei W: Novel insights into the molecular mechanisms governing Mdm2 ubiquitination and destruction. Oncotarget 2010, 1:685-690.

36. Oliner JD, Kinzler KW, Meltzer PS, George DL, Vogelstein B: Amplification of a gene encoding a p53-associated protein in human sarcomas. Nature 1992, 358:80-83.

37. Dornan D, Wertz I, Shimizu H, Arnott D, Frantz GD, Dowd P, O'Rourke K, Koeppen H, Dixit VM: The ubiquitin ligase COP1 is a critical negative regulator of p53. Nature 2004, 429:86-92.

38. Hermeking $H$, Lengauer C, Polyak K, He TC, Zhang L, Thiagalingam S, Kinzler KW, Vogelstein B: 14-3-3 sigma is a p53-regulated inhibitor of G2/M progression. Molecular cell 1997, 1:3-11.

39. Yang HY, Wen YY, Chen CH, Lozano G, Lee MH: 14-3-3 sigma positively regulates p53 and suppresses tumor growth. Molecular and cellular biology 2003, 23:7096-7107.

40. Lee MH, Lozano G: Regulation of the p53-MDM2 pathway by 14-3-3 sigma and other proteins. Semin Cancer Biol 2006, 16:225-234.

41. Chen B, Zhao R, Su CH, Linan M, Tseng C, Phan L, Fang L, Yang HY, Yang H, Wang $W$, et al: CDK inhibitor p57 (Kip2) is negatively regulated by COP9 signalosome subunit 6. Cell Cycle 2012, 11:4633-4641.

42. Lee MH, Reynisdottir I, Massague J: Cloning of p57KIP2, a cyclin-dependent kinase inhibitor with unique domain structure and tissue distribution. Genes Dev 1995, 9:639-649.

43. Lee MH, Yang HY: Negative regulators of cyclin-dependent kinases and their roles in cancers. Cell Mol Life Sci 2001, 58:1907-1922.

44. Kamura T, Hara T, Kotoshiba S, Yada M, Ishida N, Imaki H, Hatakeyama S, Nakayama K, Nakayama Kl: Degradation of p57Kip2 mediated by SCFSkp2-dependent ubiquitylation. Proc Natl Acad Sci USA 2003, 100:10231-10236

45. Zhou BP, Liao Y, Xia W, Zou Y, Spohn B, Hung MC: HER-2/neu induces p53 ubiquitination via Akt-mediated MDM2 phosphorylation. Nat Cell Biol 2001, 3:973-982.

46. Xue Y, Chen J, Choi HH, Phan L, Chou PC, Zhao R, Yang H, Santiago J, Liu M, Yeung GE, et al: HER2-Akt signaling in regulating COP9 signalsome subunit 6 and p53. Cell cycle 2012, 11:4181-4190.

47. da Silva CJ, Miranda Y, Leonard N, Ulevitch RJ: The subunit CSN6 of the COP9 signalosome is cleaved during apoptosis. J Biol Chem 2007, 282:12557-12565

48. Hetfeld BK, Peth A, Sun XM, Henklein P, Cohen GM, Dubiel W: The COP9 signalosome-mediated deneddylation is stimulated by caspases during apoptosis. Apoptosis 2008, 13:187-195.

49. Iyer SV, Iwakuma T: A novel link between the HER2-Akt and MDM2-p53 pathways via CSN6. Cell Cycle 2012, 11:4112
50. Zdzalik M, Pustelny K, Kedracka-Krok S, Huben K, Pecak A, Wladyka B, Jankowski S, Dubin A, Potempa J, Dubin G: Interaction of regulators Mdm2 and Mdmx with transcription factors p53, p63 and p73. Cell Cycle 2010, 9:4584-4591.

51. Pei D, Zhang Y, Zheng J: Regulation of p53: a collaboration between Mdm2 and Mdmx. Oncotarget 2012, 3:228-235.

doi:10.1186/1747-1028-8-14

Cite this article as: Zhang et al:: The COP9 signalosome subunit 6 (CSN6): a potential oncogene. Cell Division 2013 8:14

\section{Submit your next manuscript to BioMed Central and take full advantage of:}

- Convenient online submission

- Thorough peer review

- No space constraints or color figure charges

- Immediate publication on acceptance

- Inclusion in PubMed, CAS, Scopus and Google Scholar

- Research which is freely available for redistribution

Submit your manuscript at www.biomedcentral.com/submit
C Biomed Central 